Voix et Images

\title{
Une emprise réciproque : Clément Marchand-Alfred DesRochers (1931-1949)
}

\section{Joseph Bonenfant}

Volume 16, numéro 1 (46), automne 1990

Les correspondants littéraires d'Alfred DesRochers

URI : https://id.erudit.org/iderudit/200873ar

DOI : https://doi.org/10.7202/200873ar

Aller au sommaire du numéro

Éditeur(s)

Université du Québec à Montréal

\section{ISSN}

0318-9201 (imprimé)

1705-933X (numérique)

Découvrir la revue

\section{Citer cet article}

Bonenfant, J. (1990). Une emprise réciproque : Clément Marchand-Alfred DesRochers (1931-1949). Voix et Images, 16(1), 53-63.

https://doi.org/10.7202/200873ar d'utilisation que vous pouvez consulter en ligne.

https://apropos.erudit.org/fr/usagers/politique-dutilisation/ 


\section{Une emprise réciproque: Clément Marchand- Alfred DesRochers (1931-1949)}

\section{par Joseph Bonenfant, Université de Sherbrooke}

D'abord cet hommage d'Alfred DesRochers, un dizain vraisemblablement inédit, sans doüte de 1939:

\section{À Clément Marchand}

Que l'ennui s'abolisse aux reflets des soirs rouges Et revivent les temps où le rythme était bon À nous oblitérer les palais et les bouges!

Nous savions, à nos sots métiers, faire faux-bond;

Chacun de nous alors suivait sa trajectoire, Sans le moindre souci du bourgeois pudibond.

Aujourd'hui tu reçois le baiser de la gloire; Moi je me satisfais, restant humble grillon, D'attendre les pourpris célestes, pour y boire Avec saint Paul (Verlaine) et saint François (Villon)

Puis ce vibrant hommage, du 3 juillet 1989, que Clément Marchand m'apporte fumant, par surprise, après me l'avoir prudemment mais nettement refusé:

En relisant des lettres et des inédits d'Alfred DesRochers.

Si je ne me sentais pas humble devant l'œuvre de DesRochers - comme en témoignent les soixante-dix-huit lettres que je lui écrivis de 1931 à 1949 - je serais un monstre de naïveté et d'idiotie combinées de stupide prétention. Mais, Dieu merci, la nature m'a imparti assez de sens commun pour reconnaître sa grandeur, son génie et ramper littérairement contre ses dons éminents en face de ses hymnes et de ses odes.

J'espère pouvoir rendre hommage à ce grand poète, figure de proue de la poésie française en Amérique. Ce que je tentais de lui dire dans mes lettres est qu'il était français par son métier mais américain d'accent, poète dionysien du Nouveau Monde, même s'il développe des thèmes sortis de la mémoire atavique. 
Il était pour moi le visionnaire, le libertaire illuminé. Le souffle des espaces soulevait son chant lyrique.

Il est en effet, à mes yeux, avec Choquette, le dernier grand lyrique de la poésie française. Il daigna s'intéresser au poétereau que j'étais vers 1930 et lui accorder, en plus de son amitié qui ne se démentit jamais, une considération critique (plus généreuse que méritée) au cours d'une correspondance excessivement présente à tout ce qui touche à la poésie.

On me dira peut-être que je m'emballe facilement sur cette époque et qu'on ne peut endosser mes jugements. J'en appelle à Olivar Asselin, à Claude-Henri Grignon, à Albert Pelletier, bons juges de la qualité littéraire, pour reconnaître en DesRochers le poète le plus inspiré qu'ait produit l'Amérique française. De Nelligan à Choquette, il faut passer par $A$. D. qui les sous-tend.

Je fais souvent des blagues idiotes quand je dis qu'un DesRochers à son meilleur fait mal paraître le père Hugo. Il arrive que le disciple dépasse le maître. Cela s'est produit pour l'auteur d'À l'ombre de l'Orford vis-à-vis de certains des poètes les plus importants de fin du XIX ${ }^{e}$ et commencement du $X X^{e}$ siècle, quand il a conçu des pièces au lyrisme aussi puissant que celui de "Désespérance romantique" et de "Hymne au Vent du Nord".

\section{Cette correspondance m'a profondément marqué.}

\section{Clément Marchand}

Encore aujourd'hui, on lit, chez Marchand, la même humble déférence envers le maître, mais dépouillée des oripeaux d'une certaine naïveté apparente aux débuts de la correspondance. Quel autre exemple pourrait-on trouver d'une amitié littéraire aussi entière, d'une franchise dont on verra parfois le franc sourire, mais aussi les crocs têtus? Marchand et DesRochers se sont beaucoup entraidés comme poètes; ni l'un ni l'autre ne peut échapper entièrement au reproche d'une complaisance momentanée, mais chacun a su, à certains moments, faire preuve d'une férocité dont on n'a plus d'exemple sous les yeux, surtout dans des échanges épistolaires. La recette de cette mutuelle émulation s'est comme perdue. Quoi qu'il en soit, on voit, par ce témoignage actuel, le climat de la passion littéraire qui marque la correspondance. C'était forcé. Quand ils commencent à s'écrire en 1931, le poète de l'Estrie est déjà au faîte de la gloire; le séminariste trifluvien, même s'il a déjà écrit entre cinq et sept mille vers, n'a encore reçu aucune reconnaissance, si ce n'est de ses professeurs, dont Eddie Hamelin, et de son préfet des études, l'abbé Albert Tessier, son premier père en poésie. Le débat sourd qui va marquer les échanges, c'est cette différence entre un poète qui a fait 
trois ans d'études classiques, et un autre, qui en aura bientôt complété tout le cours. L'un est instruit, l'autre ne l'est guère, même s'il lit énormément. Ils ne lisent pas les mêmes choses: Marchand lit les Français; DesRochers lit les Américains; il le fait même parce qu'il veut apprendre quelque chose. Dans cette différence de scolarisation et de renommée repose l'origine de l'ascendant que chacun exercera sur l'autre. L'un a 18 ans; l'autre, 30. DesRochers devra piaffer énergiquement pour mettre l'aspirant poète au pas, et au bon.

La précocité du jeune Marchand est certes le phénomène le plus frappant des débuts de la correspondance. Le raccourci serait indu si on affirmait que ces lettres tournent autour d'une théorie de la poésie; l'anachronisme, de plus, étonnerait. Mais on ne se trompe pas si on voit, au centre de toutes les lettres, la question de la PRATIQUE DE LA POÉSIE. L'une est près de l'autre, mais il faut tenir à PRATIQUE et à TECHNIQUE, car ici ce sont des poètes, et non des théoriciens, qui parlent à perte de vue de ce que peut et doit être la poésie.

Quand, dans sa première lettre (24 janvier 1931; Cher Monsieur DesRochers), le pauvre collégien hésite à dire son admiration pour l'auteur de l'Orford, devenu son livre de chevet, il ne lui dit pas qu'il a déjà à son actif plus de cinq mille vers. Il garde un bon souvenir du poète qu'il a rencontré à la réception de décembre 1930 chez les franciscains. Celui qui écrit: Je n'ai d'autre expérience que celle de mes dix-huit ans ne révèle pas tout de suite qu'il prépare déjà les Soirs rouges. Mais il lui en parlera tout au long de l'année 1931, et jusqu'en 1939, année de leur publication. J'écris les pièces de Soirs rouges spécialement pour vous, écrira-t-il plus tard. Et encore: Je suis, en ces temps de chômage, à bredouiller les Soirs rouges avec difficulté. (6 novembre 1931)

Les lettres de 1931 montrent le réseau d'idées et d'émotions qui se tisse entre les deux poètes, l'aide réciproque qu'ils s'apportent, la confiance qu'ils se témoignent, la connaissance qu'ils acquièrent l'un de l'autre, et l'un par l'autre. D'abord la passion de la poésie. Marchand écrit: Depuis que j'ai commis ma première rime, je ne me suis plus appartenu. Des vers! Je rêve d'eux la nuit, j'en fais en me levant, ce qui est pis - sans le vouloir - j'en rumine jusqu'aux offices divins. Il lui demande comment corriger ses défauts les plus fâcheux et va jusqu'à se traiter de rimailleur. Devant les éloges du maître, il se rebiffe. Vous êtes un excessif. Je ne vous connais pas de milieu. Mais de grâce ne me prenez pas tellement au sérieux. Songez que je n'ai pas encore le nombril sec. Plus tard, il reconnaît recevoir de lui un exemple de condensation.

Entre élan et retenue, c'est une amitié intellectuelle qui s'établit sur la base de l'admiration. Mais comment ne pas décevoir? Chose certaine, Marchand a voué dès le début une admiration entière à 
DesRochers. Il lui avoue qu'il s'est mis à rimer pour supporter la vie dure et prosaïque que l'on s'ingénie à créer autour des orphelins. Il trouve en lui quelqu'un pour l'écouter. Et il généralise (heureusement) son cas: J'estime qu'en littérature, jeunes et aînés doivent marcher main dans la main. À cette fraternité, les jeunes y gagnent en maturité, et les aînés, en perpétuelle verdeur. (25 février 1931) Celui en qui il reconnaît un chef de file $(24$ mars) et notre meilleur poète au Canada (2 avril), il voudrait en faire son frère intellectuel. C'est à cela que je tends. Je promets de travailler et de suivre en autant que je puis, votre sage directive. Je relis pour la centième fois vos lettres. Déchirez-moi ce brouillon électrique. $N$ 'y répondez pas. C'est perdre du temps. (10 mai) La demande fraternelle est forte, mais ne va pas sans rejet. Quand il apprend que DesRochers lit de ses vers autour de lui, il s'écrie: Vous êtes un père pour moi; mais il se ravise immédiatement en ajoutant: Il y a de la fraternité dans ce que vous faites pour moi. (10 juillet) Comment le jeune poète n'aurait-il pas eu cette admiration pour celui que Nérée Beauchemin mettait sur un pied d'égalité avec les meilleurs aigles du parnassisme français. Il m'a dit qu'il mourrait content, ayant vu l'aurore d'une nouvelle ère en poésie. (18 mai 1931) On peut admirer dans cette correspondance une acceptation mutuelle entière, ce qui d'ailleurs n'empêchera jamais les critiques et les reproches même les plus acerbes. Je voudrais être à la hauteur de l'opinion que vous avez de moi (10 mai): voilà bien une phrase qui fait penser à l'amitié de Montaigne pour la Boétie et que DesRochers aussi aurait pu écrire, mais il ne l'a jamais fait.

Dans ses vingt-six lettres de 1931, Marchand, qui veut affermir sa technique pratique du vers (24 mars), établit un sorte d'équivalence entre la robustesse de DesRochers et ce que doit être le vers français. Il affectionne le lyrisme de bonne santé et la puissance d'émotivité de son correspondant. Il parle de la vertu stimulante de leur commerce épistolaire. Donnez dans le robuste, lui écrit-il, rien d'autre ne vous convient. Votre cerveau est puissant. Il vous faut retourner à la manière de l'Orford. (25 mai) Il lui écrit, de SainteGeneviève de Batiscan où il travaille sur la ferme d'un oncle: J'aimerais vous voir ici, mon cher DesRochers, avec votre fort tempérament de poète-bûcheron. Que ne venez-vous passer une semaine avec moi! (10 juillet) Il verra quelques jours plus tard à Sherbrooke un bonhomme tumultueux. C'est toujours cette force, cette puissance; que Marchand demandera à DesRochers; c'est en son nom qu'il lui assènera parfois de vifs reproches; il veut une poésie humaine, on oserait dire élémentaire, proche des gens. Marchand sera le poète des citadins-prolétaires et le grand nouvelliste de la vie rurale ancestrale, on le sait. Il invite DesRochers à se rapprocher du peuple:

Le peuple est une énigme que nul n'a pu percer à distance. Qui pourrait parler de lui sinon lui-même? Il faut vivre auprès de 
lui, l'aimer, entrer dans l'intime de sa vie, pour pouvoir en parler. Or je n'ai pas vécu avec le peuple. Je ne l'ai côtoyé qu'accidentellement. Donc le poète peuple, c'est toi qui le seras. (6 novembre)

On voit donc s'établir entre les deux poètes une amitié qui joue sur plusieurs plans: intellectuel, littéraire, social, mais aussi théorique, au sens où la théorie est à la fois regard et prise de position sur les questions essentielles, mais aussi regard essentiel sur la vie, l'amitié, la poésie, l'amour, la misère (nous sommes alors en pleine crise économique) et autres misères, celles-là quotidiennes. Pour mieux voir comment cette belle amitié humaine s'est formée, il importe de relire les premières lettres de DesRochers au jeune poète de Trois-Rivières.

Le moins qu'on puisse dire, c'est qu'il n'a pas levé le nez sur l'hommage qui lui parvint d'un inconnu en cette fin de janvier 1931. De la part de celui qui correspondait déjà avec Émile Coderre, Simone Routier, Éva Senécal, Alice Lemieux, Robert Choquette, Louis Dantin, Rosaire Dion, Albert Pelletier, Harry Bernard, Lucien Rainier et Claude-Henri Grignon, on aurait pu s'attendre à au moins de la tiédeur. Pas du tout. Nous sommes tous un peu vaniteux, écrit-il (27 janvier 1931), et ce nous fait «un p'tit velours» d'être si considérés. Puis il nomme huit poètes, dont Émile Nelligan, René Chopin et Paul Morin à qui il doit infiniment, et d'autres qui comptent aussi, dont Octave Crémazie, William Chapman et Eudore Évanturel. Enfin, les fleurs, enivrantes: Je vous parle de vos vers. Ils sont supérieurs et je n'hésiterais pas à les signer. Vous avez une harmonie, Monsieur! Vos vers sortent tellement de la production courante que je les croyais d'un auteur français. Pour finir, une phrase-thème qui parcourra toute la correspondance, énoncée clairement, comme un avertissement: Le poème en prose m'a toujours semblé un genre bâtard. Avis!

Dans sa deuxième lettre (7 février 1931), après avoir complété son palmarès avec Nérée Beauchemin (plus riche que Paul Morin, parce que plus égal), le premier mentor littéraire de Marchand, DesRochers se lance dans l'éloge de sa race familiale:

Je descends d'une lignée de quinze générations de défricheurs (le premier. Houde - dit des Rochers - s'en vint à l'île d'Orléans en 1642). Mon bisaieul a arpenté tout le continent à peu près, a marié une montagnaise et ouvert quatre terres; mon grand-père en a ouvert deux, avant de revenir au "bien paternel»; mon père a colonisé trois terres, avant de mourir à la peine, à 47 ans. J'ai un frère qui a ouvert deux terres... Le sens du réel, nul ne l'a plus que nos paysans. Pourquoi, dès qu'on sait lire, le perd-on, ce sens du réel? Je ne l'ai jamais compris.

L'auteur de l'Orford transmet au futur auteur de Courriers des villages une énergie et une vịsion grandioses. Mais dès la quatrième lettre (28 février 1931), le magister pointe le bout de l'oreille: Vous 
êtes un poète complet: vou's n'avez plus qu'à apprendre votre métier à fond. Il l'assure qu'il promet autant que Choquette, mais voit aussi le même danger: Vous méprisez trop les règles de la prosodie. Voilà un leitmotiv, déjà. Et un acte d'humilité: Je n'ai que des études incomplètes et il me manque la belle discipline de l'ordre, de l'agencement logique. Et je vous envie d'avoir fait des études complètes.

Après ces premiers échanges pacifiques, les lettres vont parfois s'enflammer. On s'échange quantité de poèmes pour lecture et correction. Le 18 avril, Marchand signale cinq qui ou que dans une strophe de DesRochers. Ce dernier en fut-il chatouillé? Toujours est-il que dans sa réponse du 22 avril, le Sherbrookois hausse le ton: Si votre tempérament vous pousse à chanter les oreilles en poussière dè Toutankh Ammon, chantez-les, et vous serez plus dans l'ordre qu'à chanter les pagées de clôture de Berthier-en-Haut. Il écrit au passage: Votre photo révèle à peine un léger duvet, et ajoute: Vos sonnets, il faut qu'ils soient FAITS, ou bien je vous eng...uirlande. Au début de mai, Marchand hurle une lettre furieuse contre lui-même:

Votre coup de poing me fait hurler de rage contre moi, qui suis la bêtise incarnée. Si après les efforts que j’ai fournis [il lui a révélé le 30 avril qu'il a écrit 7000 vers de débutant], j'en suis au niveau de Boulanger, c'est à croire que je n'irai pas plus haut. Je vais être une décade sans téter les Muses. Je n'ai commis que des fiascos. Mes possibilités sont celles d'un mortné. Je vous remercie de votre franchise.

Le $8 \mathrm{mai}$, DesRochers lui répond sereinement et lui redonne confiance en lui-même: Vous m'avez fait rire à m'en décrocher les mâchoires, en m'annonçant que vous seriez une décade sans téter les Muses. Le 10 mai, repentance du jeune poète:

Aujourd'hui, je suis un gars canadien sans lâcheté, sans forfanterie. J'ai honte du billet que je vous ai jeté l'autre soir, avec quelle crânerie déplacée. Déchirez ça sous vos semelles, pas avec vos mains. Je fus lâche au cube de vous dire que j'avais l'idée de me laisser mourir stupidement. Vous savez, je suis un impulsif de la pire espèce. La rage et l'enthousiasme se partagent le meilleur de moi-même, etc.

À partir de ce moment, la liberté de ton est trouvée. Toute la correspondance est marquée par une entreprise intellectuelle réciproque qui ne restera étrangère à aucune dimension littéraire, socio-culturelle, et même humaine, même existentielle, dans ce qu'elle comporte de plus empirique. S'écrire aura la même valeur que se taire; tarabuster l'autre ne sera pas plus grave que l'encenser. DesRochers écrit le 2 janvier 1933:

Quant à mon royal silence, je ne vois pas que tu aies plus à t'en plaindre que moi du tien. Je m'intéressais vivement à ton 
talent quand je pouvais têtre de quelque utilité; depuis assez longtemps, il semble bien que tu n'aies plus besoin de mes conseils. Tu es parfaitement libre d'écrire comme tu voudras.

Il est vrai que, dès l'été 1932 , Marchand travaille corps et âme au Bien public; il a moins de temps, mais il écrit toujours; les Soirs rouges sont toujours sur le métier. Et DesRochers lui ramène son leitmotiv:

Je trouve que tu perds ton temps complètement et que tu gaspilles ton talent, en essayant de faire du vers libre sur des sujets de terroir. C'est aux antipodes! Le vers libre est une invention moderne qui doit servir à traduire le désarroi moderne. Un seul instrument convient à toutes les musiques: le piano, et son équivalent en littérature: le vers régulier.

Il va même jusqu'à écrire, le 20 janvier 1933: Employer le vers libre comme l'emploient Claudel et consorts, c'est une abomination; on ne dit pas la messe dans un bordel! Quelques mois plus tard, après la cuite de Québec, qui lui a valu de la part de Marchand de vertes remontrances (Maudit scotch!'Je suis un crétin, un cochon, un trou de..., s'excuse DesRochers, honteux), on voit, à une remarque de ce dernier, que le vers classique n'est pas qu'une-affaire de théorie; c'est une figure et une quête de sagesse, un moyen de discipliner sa vie: Mon cuvre publiée ne vaut absolument rien du tout. Je me rends compte qu'à moins de rétablir la technique parnassienne à ma vie de tous les jours, tous mes beaux projets sont finis. Il projette même une retraite fermée chez les franciscains à Chateauguay pour y rencontrer "le père Marcel». A partir de ce moment, les lettres vont s'espacer autant que vont se multiplier les rencontres des écrivains, à Sherbrooke, à Trois-Rivières ou à Montréal. L'amitié survit intégralement, en dépit des aléas de l'existence. Comme poètes, Marchand et DesRochers s'entrecorrigent, s'interstimulent; comme journalistes, ils se réclament mutuellement des textes. Chacun sait bien défendre, et protéger, sa pratique. Ainsi, DesRochers, le 2 janvier 1938:

Tu me demandes pourquoi j'écris des sonnets quand j'aic'est toi qui le dis! - un souffle de bœuf. Le sonnet, avec son cadre précis, quand on a le temps de le travailler, convient à tous les thèmes. Et si un seul est trop étroit pour tout enclore, on série ses pensées et l'on construit une série de sonnets.

Les lettres abondent en remarques du genre, en conseils, en émulation. DesRochers a même imité Marchand dans un poème populiste dont voici le premier quatrain:

Dans la crasseuse odeur qui monte des usines,

Ma chair hume un relent fraternel de sueurs

Et parmi le fracas étourdi des machines

J'écoute des chansons défuntes de bonheur 
Il ajoute, en post-scriptum, au bord de l'ironie amicale: Voici le premier jet d'un poème dont tu es le père, puisque c'est à lire les tiens que l'idée m'en est venue. Temps de composition: exactement 47 minutes, sans retouche, au Remington. Le poème comprenait en tout trente-cinq vers. DesRochers n'a pas persisté dans cette veine.

Pour revenir aux lettres de Marchand, disons qu'il y a une coupure en 1932. Avant cette date, on a droit aux lectures du collégien (Dimmet, Georges Valois, Le Franc, Constantin-Weyer, Routier, mais aussi à la philosophie thomiste, etc.) (bonum et ens convertuntur). Tes opinions arrivent à conflagration immédiate avec les données de saint Thomas, lui écrit-il le 7 février, à lui qui avait affirmé: L'œuvre immorale est l'œuvre mal écrite. Tanné d'arroser des fleurs dans une serre-chaude, ne pouvant 'devenir cultivateur, pour des raisons pécuniaires, ni soudeur dans la machine-shop de son oncle à Montréal, Marchand entre au Bien public en juillet 1932, et dans les nécessités de la vie, dont le mariage en 1935. Le $1^{\mathrm{er}}$ septembre 1936, il déplore ne pas trouver moyen de se raccorder $\grave{a}$ la littérature et à ses connexités. La chienne de vie est bien dure à gagner. L'année 1937 prouve que l'admiration ne baisse pas. 10 juillet: Cher et grand divin, ami des classes pauvres et sans doute assez pauvre toi-même en écus quoique doté d'un fantastique esprit d'entreprise; 11 décembre: Tu es actuellement le seul en terre d'Amérique à pouvoir écrire des vers d'une frappe précise et dure qui me font penser à du Moréas et d'un lyrisme aussi pur que celui de Valéry dans Charmes; et, le 29 décembre, une idée étonnamment moderne: C'est mon avis que tout se résout à la forme. Il n'y a d'ailleurs dans la vie que des formes.

Ce même 29 décembre 1937, quelques lignes emportées expriment une fois pour toutés, peut-on dire, la déception que Marchand a souvent manifestée en regard de la productivité réduite de DesRochers:

Je crois que tu bois trop, espèce de cochon. Si tu crèves sans avoir donné à ton pays les chefs-d'œuvre qu'il est en droit d'attendre de ton magnifique talent, tu peux être sûr que je n'irai pas à tes funérailles. Mais pendant qu'on enterrera ta carcasse, je consacrerai un éreintement soigné à ton œuvre. Ce sera ma façon de te manifester mon admiration déçue.

(J'étais son garde-fou. Il avait besoin de mon soutien moral. J'étais sa lampe allumée. Il me craignait. ${ }^{1}$ ). Un autre moment de déception, cette pierre de touche des amours vrais et des amitiés authentiques, surgira en 1948: Sans toi, je n'eus rien été. Il m'a souvent semblé que je n'étais plus digne de la considération que tu as prodiguée à mes premiers essais. (13 janvier) J'ai écrit ça! se surprendra-t-il en 1989. Mais il a aussi écrit, le $1^{\mathrm{er}}$ avril 1942 , avec une assurance à faire blêmir quiconque: Les poétereaux de l'amphigourisme, les imitateurs

1 Rencontres avec Clément Marchand, les 30 juin et 3 juillet 1989, à Trois-Rivières. 
de Valéry... Ce ne sont plus que jeux dans l'espace et songes en équilibre, que voltiges dans un vent de folie. En 1948, Marchand n'est pas loin d'être désillusionné. Il envie DesRochers de n'avoir pas sombré dans la détestation, le doute et la misanthropie. Lui se compte pour mort à la littérature (13 janvier). À un moment donné, tu crèves d'indigestion de tout ce que tu respires. Ses lettres prennent graduellement fin sur un constat d'apaisement, on dirait d'assagissement:

Depuis quelques années déjà, j’ai dépassé le stade d'infantilisme où le débutant recherche l'encens et la louange. Je cherche avant tout à savoir si je vaux quelque chose. J'ai perdu ma belle assurance. Je n'ai pas écrit un chef-d'œuvre [il fait allusion aux Soirs rouges; il a trouvé l'accueil de DesRochers tiède]. À la bonne heure pour moi, c'est tout ce dont j'ai besoin. (22 mars 1948)

La corrèspondance prend fin peu à peu de cette manière un peu mélancolique.

Il ne faut pas, aujourd'hui, s'étonner de cette tournure finale, étant donné que les passions littéraires ne sont plus ce qu'elles étaient. À l'époque, on savait critiquer aussi fort et atroce in præsentia qu'in absentia. On a l'impression que dans les années trente et quarante, les critiques positives s'additionnaient et que les critiques négatives s'annulaient. Quand on a eu, comme Marchand, des correspondants aussi prestigieux, et variés, que Nérée Beauchemin, Albert Pelletier, Harry Bernard, Raymond Douville, Olivar Asselin, Claude-Henri Grignon, Ulric Gingras, Paul Morin, Robert Choquette, François Hertel, Jacques Ferron, Albert Tessier, Léo Ayotte, Albert Lévesque, Simone Routier et Sarah Larkin (poète américaine), on ne peut qu'être devenu prudent sur ce que signifie l'amitié entre écrivains. L'amitié Marchand-DesRochers est avant tout franche et virile. Marchand l'a bien caractérisée le 14 mars 1939 quand il projetait de dédier les Soirs rouges à DesRochers; mais il ne l'a pas fait, craignant de le décevoir. Il y pensait en signe d'une robuste amitié au-dessus du temps et de l'espace. Telle nous paraît-elle aussi à nous, une amitié qui, au début, s'est rapidement dépêtrée des formules de politesse pour parcourir tous les hauts et les bas, les espérances et les déceptions possibles.

Nulle amitié ne fut plus fraternelle, étant donné l'origine terrienne commune. Je descends, dit Marchand, d'une gent de charrue et de bêche qui n'avait de lettres que les brochures agricoles, les annales et les almanachs. ${ }^{2}$ Mais si elle ne fut pas réticente, cette amitié fut assurément clairvoyante. Rappelons deux écrits de DesRochers hors correspondance. Le premier, en 1932:

2 "Réponse de Clément Marchand" à l'allocution de l'abbé Albert Tessier, Société royale du Canada (section française), $n^{\circ} 5,1947-1948$, p. 60. 
Une chose m'inquiète au sujet de Marchand: c'est son inclination pour le vers libre [...] En dessous des théories littéraires $d u$ vers libre, il y a des théories sociales, joserais dire des théories morales, qui ne sont pas celles de Marchand. [...] Clément Marchand pourrait bien être le vrai poète que le peuple canadien-français attend depuis sa naissance. Il a le souffle lyrique et le don poétique. Il lui manque la discipline personnelle. J'espère que l'âge la lui enseignera. ${ }^{3}$

Le second, en 1938. DesRochers y nomme les prosateurs contemporains marquants: Albert Pelletier, Claude-Henri Grignon, et peut-être celui dont l'influence sera la plus profonde, s'il trouve un thème à la hauteur de son talent natif - Clément Marchand. ${ }^{4}$ Le doute a donc persisté, sinon l'espérance, autant dire la déception.

Marchand a bien vu sa relation avec DesRochers: Il a eu la patience de potasser ses premiers grimoires. Nous avons été de francs amis qui, le plus souvent, avouions les mêmes admirations. ${ }^{5}$ Il a bien saisi sa visée poétique centrale: Pour lui, rien n'importait autant qu'un classicisme de fond habillé de mille formes modernes. ${ }^{6}$ Mais il n'a pas manqué d'humilité en affirmant: Nous savons que DesRochers, s'il est complaisant pour les œuvres des autres, est très difficile pour les siennes. ${ }^{7} \mathrm{Il}$ a eu aussi le courage, du vivant de DesRochers, mais onze ans après la fin de la correspondance, de dire sa conviction profonde face aux oukases de DesRochers contre le vers libre, et contre tout ce qui ne se conformait pas à la technique parnassienne; il n'a pas pu ne pas penser à son vieux maître dans sa conférence sur les "Tendances de la jeune poésie au Canada français":

Quant aux techniques à employer, nous nous gardons bien d'approuver l'exclusive décrétée contre tel ou tel genre. Vers réguliers ou VERS LIBRES, poésie entièrement libérée, [...] peu importe la forme choisie pourvu qu'elle corresponde à une ascèse impérieuse et que son mouvement obéisse à un rythme intérieur. ${ }^{8}$

Marchand, incontestablement, est un écrivain plus ouvert, plus polyvalent que DesRochers. La faveur enthousiaste dont il jouit, en cette décennie, dans tous les milieux littéraires québécois, n'en estelle pas la preuve irréfutable?

Celui qui écrivait froidement à DesRochers: Ne craignez pas de me froisser (25 février 1931), ou encore: Taisez donc toute louange à

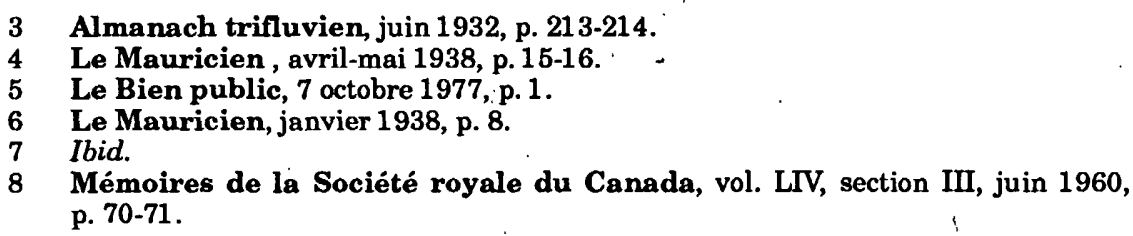

8 Mémoires de la Société royale du Canada, vol. LIV, section III, juin 1960, p. 70-71. 
mon égard (24 mars 1931), ne pouvait prendre qu'avec un grain de sel les paroles souvent trop louangeuses de son mentor, de même que, plus tard, l'avalanche de prix littéraires qui s'est abattue sur ses œuvres. Pourtant, celui qui avait vu un de ses sonnets publié dans le Devoir (à 15 ans!) et qui avait récolté en 1930 (en Rhétorique, à 18 ans!) son premier laurier littéraire, le prix Parker, disputé entre les collèges classiques affiliés à Laval ${ }^{9}$ avait fait preuve d'une précocité hors du commun. Dès ses premières lettres à DesRochers, en 1931, on s'étonne de la maîtrise de la phrase et de la syntaxe françaises, de la richesse et de la justesse du vocabulaire, de l'étendue de la culture, de la netteté et de l'opiniâtreté des idées et des partis pris. Mais surtout, qu'un jeune Québécois se soit déjà, à cet âge, tant et si sûrement passionné pour la littérature de son pays, est pour nous un sujet de réflexion et un objet d'admiration que le temps ne pourra jamais abolir. On ne s'étonne pas que Marchand ait exercé, et exerce encore sur tant d'écrivains, dont DesRochers, un ascendant dont on ne peut se défendre. Cela aussi, Marchand le voit lucidement. DesRochers a été influencé par son disciple plus qu'on ne le croit. J'ai influencé DesRochers même dans la poésie. Si on s'était vus plus souvent, $j$ 'en aurais fait un autre poète. ${ }^{10}$

Le grand paradoxe de l'œuvre de Marchand et de DesRochers, à travers le prisme de leur correspondance, c'est celui de la rupture avec la tradition poétique française ${ }^{11}$. Leur éternel conflit, en dépit de tout, porte sur l'innovation des formes. La marotte parnassienne de DesRochers était à la fois une ligne sûre et une provocation salutaire: ne peut-on aujourd'hui la voir comme une idée de contrainte, qui en vaut d'autres, dites modernes? Une technique inlassablement préconisée et martelée amène forcément ses tenants à un point de rupture. Dans les Soirs rouges, Courriers des villages, dans $\ddot{\mathbf{A}}$ l'ombre de l'Orford, on voit cette contrainte, on entre dans cette rupture, on reçoit de l'énergie, on éprouve cette novation de toutes choses. La poésie a trouvé son lieu, et le paradoxe, son dénouement.

9 "Présentation de Clément Marchand par l'abbé Albert Tessier", Société royale du Canada, loc. cit., p. 53-54.

10 Interview inédite de Clément Marchand à Richard Giguère et à Dominique Garand, le 22 mars 1985.

11 Sur la rupture de DesRochers avec la tradition française, en dépit de la technique parnassienne, sur son "énergie lyrique", et sur la création de la liberté sauvage du métis DesRochers, lire l'article de Claude Filteau, "Rhétorique et philosophie politique. De la cité antique à l'idée de patrie, chez Jean Charbonneau et Alfred DesRochers ", Voix \& images, ${ }^{\circ} 41$, hiver 1989, p. 250-268. 